Meta

Journal des tradlucteurs

Translators' Journal

\title{
Pour traduire la poésie : notes et notations
}

\section{Teodoro Sáez Hermosilla}

Volume 35, numéro 3, septembre 1990

La traduction dans le monde hispanolusophone

URI : https://id.erudit.org/iderudit/003069ar

DOI : https://doi.org/10.7202/003069ar

Aller au sommaire du numéro

Éditeur(s)

Les Presses de l'Université de Montréal

ISSN

0026-0452 (imprimé)

1492-1421 (numérique)

Découvrir la revue

Citer cet article

Sáez Hermosilla, T. (1990). Pour traduire la poésie : notes et notations. Meta, 35(3), 615-624. https://doi.org/10.7202/003069ar d'utilisation que vous pouvez consulter en ligne.

https://apropos.erudit.org/fr/usagers/politique-dutilisation/ 


\section{POUR TRADUIRE LA POÉSIE : NOTES ET NOTATIONS}

TEOdORO SÁEZ HERMOSILLA

Espagne

Une théorie de la traduction poétique n'est pas à coup sûr une entreprise réalisable à court terme, les acquis dans ce domaine de la traductologie étant encore embryonnaires ou peu s'en faut. Mais nous avons au moins deviné la seule voie qui pourra nous y conduire: la recherche parcellaire, un travail qui doit se mener à travers les poétiques concrètes des langues concrètes. Autant dire que la traduction poétique doit être avant tout une opération poétique, comme le voulait Edmond Cary pour qui la linguistique, que ce soit la générale, ou celle que l'on prétend appliquer comme panacée dans tous les phénomènes qui relèvent directement ou indirectement du langage, est, de toute évidence, insuffisante.

Contentons-nous donc pour l'instant de mettre en ouvre un travail d'approfondissement sur certains aspects ou paramètres des textes poétiques dans le dessein d'assurer leurs fondations les plus adéquates, même si l'érection de la charpente d'un aussi complexe, aussi beau et aussi étrange édifice, en a encore pour un bon moment.

Ce n'est certainement pas la première fois que je me mets à cette difficile - et si noble - tâche, les quelques réflexions que j'offre par la suite étant le résultat d'une démarche tâtonnante dans la confrontation de deux langues sœurs dont le français constitue la langue poétique de départ et l'espagnol, celle d'arrivée.

Ce que j'ai fait préalablement avec des poèmes de Baudelaire, de Verlaine, de Rimbaud, de Mallarmé, de P. Valéry et de G. Apollinaire, de P. Éluard et de R. Char fera place ici à un poète plus actuel, Yves Bonnefoy, qui prolonge toute cette modernité-là dans la quête du véritable phénomène de la démarche poétique.

Il n'y a qu'à choisir, en suivant l'intuition et un certain savoir-faire, un poème dont la valeur et la réussite soient difficilement contestables. Tel peut être le cas de La Beauté, dont voici le texte:

Celle qui ruine l'être, la beauté,

Sera suppliciée, mise à la roue,

Déshonorée, dite coupable, faite sang

Et cri, et nuit, de toute joie dépossédée

$\hat{O}$ déchirée sur toutes grilles d'avant l'aube

Ô piétinée sur toute route et traversée,

Notre haut désespoir sera que tu vives,

Notre cour que tu souffres, notre voix

De t'humilier parmi tes larmes, de te dire

La menteuse, la pourvoyeuse du ciel noir,

Notre désir pourtant étant ton corps infirme,

Notre pitié ce cour menant à toute boue ${ }^{1}$.

Ce n'est pas précisément la première fois qu'un tel sujet a été traité par un poète. On se rappelle immédiatement le fameux sonnet de Baudelaire du même titre dont la composition de Bonnefoy semble être à première vue le négatif ou la négation. Les deux 
textes constituent avant tout des traductions intralinguales sur le mode poétique d'une idée relevant des sciences sociales, d'une opinion du domaine public qui n'est pourtant pas vérifiable par aucun moyen démonstratif, mais dont chaque individu et chaque société se font successivement une représentation.

Il semble aller de soi que les auteurs ont incarné l'«idée» — ou brouillon senti, inspiré et conçu - en des façons de dire différentes, voire contraires, dans un accord intime avec leurs styles, leurs apprentissages et leurs jugements de valeur sur le fait poétique. Et il ne serait pas moins manifeste que l'élément premier, ce qui intéresserait en premier lieu le lecteur ordinaire (en opposition aux intérêts du critique spécialisé, du poète lecteur ou de l'érudit) serait de savoir ce que l'écrivain a voulu dire, le style et la structure formelle demeurant pour lui quelque chose de secondaire, une espèce de but second qu'il faudrait déchiffrer d'après un savoir-faire ou conformément au rituel d'un code qu'on possède ou pas et qui doit être en tout cas appliqué.

Nous ne pouvons penser que le poème soit uniquement un vouloir-dire car il incorpore une mise en œuvre, donc une expression idiomatique, idiolectique et générique (au sens du genre littéraire), mais plutôt qu'il est le lieu de rencontre d'un objet conceptuel «surdéterminé» par un autre objet qu'on peut qualifier de poématique. Le fait que le poète ait été amené à sa création indifféremment par le concept ou par la langue ne signifie pas que le lecteur ordinaire ne s'intéresse pas tout d'abord au message «idéatique» et, après, à la forme.

D'autre part, et cela révèle l'importance décisive du destinataire dans les actes de parole écrite ou parlée, une lecture adéquate supposerait la captation du contenu conceptuel uniquement à travers le déchiffrement des clés formelles. En tout cas le fait décisif est qu'objet conceptuel et objet poétique doivent s'intégrer dans un «percept» total qui se décrit dans une première approche, comme ce que le traducteur saisit grâce à un processus d'incursions interprétatives confirmées ou niées qui le dévoilent en même temps qu'elles le recréent dans la plongée formelle.

L'important est de ne jamais considérer un texte comme quelque chose d'achevé qu'on puisse démonter, «taxonomiser», mais bien plutôt comme une invitation à l'acte interprétatif qui sera le seul à pouvoir reprendre les moments presque toujours provisoires de la création dans une recréation qui lui est nécessaire pour sa propre survie comme acte de parole et, partant, comme dialogue.

La conformation d'un vouloir-dire ne devrait jamais être tenue pour quelque chose de définitif si l'on ne veut pas condamner le texte à n'être qu'un objet de sciences expérimentales, pour ne pas dire exactes, ce qui n'est pas le cas. Le texte en tant qu'acte de parole écrit ne doit pas nous tromper, de par sa fixation qui n'est qu'illusoire, sur sa fonction d'intuition radicalement communicable.

Il n'est pas un objet mais un projet, une trouvaille de parole, une mise en scène qui peut se re-dire, se représenter, se formuler indéfiniment. L'auteur y a eu accès par des à peu près de conformations préalables que le lecteur continuera de repenser et de redire. Quand ce lecteur devient traducteur, la redite sera forcément différente, parce que la nouvelle idiomaticité sera différente mais on ne voit pas en quoi ou pourquoi le caractère et la démarche de cette formulation nouvelle devraient être essentiellement différents des reformulations intralinguales pratiquées par l'auteur.

Aussi ne faut-il pas considérer le poème en marge des autres faits d'écriture comme une fin en soi, puisque tout en étant acte de parole, il se constitue sujet de dialogues, sauf si on continue à envisager la traduction comme une simple comparaison philologique portant sur la Langue, (mais on a largement démontré que la traduction comme processus instaure ce phénomène dans toute son authenticité et rentabilité scientifiques), invitation à la connaissance, recherche d'un co-auteur. 
On ne saurait dire en conséquence que la traduction poétique est impossible parce que le texte est irrépétable, sinon que ce texte est toujours susceptible d'une reprise par une quelconque volonté de réplique. Qui plus est, ce texte n'existera, si ce n'est comme matérialité faite de traits sur le papier, qu'aux moments de ses lectures grâce à ses repensées et à ses pénétrations par autrui.

Il est indispensable qu'il y ait une séparabilité entre le projet et la forme idiomatique : toute forme est idiomatique, entre le contenu conceptuel-poétique et sa conformation langagière, et cela non pas pour que la traduction soit possible mais parce que la traduction n'est pas autre chose que la manière privilégiée de la compréhension et de la réécriture.

Le fait, répété, de la reprise d'un sujet comme celui de la beauté ne fait que parler de traduction intralinguale (et/ou interlinguale) et par là du véritable caractère substantiellement récidivant et opiniâtrement divergent des sciences sociales.

La traduction se révèle comme recréation dans sa modalité de fait intralingual et se présente comme une recréation de second degré, pour ainsi dire, dans son aspect d'interlinguale, dans la mesure où elle implique une double réfraction du percept total du message de parole traditionnel.

D'un côté et tout d'abord le lecteur critique qu'est tout traducteur doit traduire intralingualement avec tout ce que cela comporte. Après il lui faut donner ce percept structuré dans et à travers l'idiome et l'idiolecte d'un texte d'arrivée.

La différence par rapport à ce phénomène universel du processus langagier qu'est la traduction intralinguale consiste en ceci, qu'on demande à ce médiateur intéridiomatique d'être toujours fidèle à ce percept compris et senti, et le paradoxe est que cette fidélité est compromise, au double processus de l'interprétation, qui ne finit pas d'en venir à bout, et de la reformulation, qui s'en écarte irrémédiablement.

Mais il est temps déjà, après ces premières «notes» théoriques qui me paraissaient nécessaires avant d'arriver aux empirismes rythmiques, de s'attaquer, ne fût-ce que succinctement, à certaines notions qui, pour avoir été introduites, ont besoin d'une justification. Et tout d'abord notre conception du SENS, ce terme que bien des critiques emploient sans pour autant justifier ou expliquer leur vouloir-dire. J'ai toujours entendu par SENS, suivant en partie les thèses des herméneutes ainsi que des Interprètes de l'École (ESIT) de Paris², le percept à la fois psychologique, social et générique que le traducteur induit par son activité exégétique.

Ce percept embrasse les contenus conceptuels en même temps que les contenus proprement poétiques et les conformations en fait de parole. Le processus de l'interprétation se développerait comme suit: dès que les ondes visuelles (ou auditives), devenues courant neuronal, sont captées et retenues par et dans la mémoire immédiate et qu'un ordinateur de notre cerveau les transforme en entité langagière compulsée et comprise, un embryon de percept se produit, objet de la conscience, qui croît et s'affirme comme un continuum que des hypothèses orientent et concrétisent au fur et à mesure.

Il y a dans l'activité herméneutique, d'une part, grâce à la mémoire à court terme, les apports du déjà-compris parce que vérifié, et d'autre part, toutes sortes de données nécessaires pour chaque «intervention» fournies par la mémoire permanente. Cependant la vérification est sujette à caution jusqu'à ce qu'on ait réalisé toutes les démarches pertinentes, jusqu'à ce qu'on ait appliqué tous les registres du savoir qui se constituent et doivent se combiner pour le texte, bref, jusqu'à ce qu'on ait étudié et parcouru séquence par séquence, mot par mot, ictus par ictus, le poème en question.

Cela fait, il demeure que nous n'aurons qu'une «solution» chimique, qu'un cadavre artistique désarticulé selon un réseau d'hypothèses qui n'appartiennent qu'à nous seuls, puisqu'elles dépendent de nos savoirs et de nos appréciations. Le vouloir exprimer du 
poète reste au double fond confondu d'une volonté de dire et une expérience de création réalisée insaisissables dans ce que cela comporte d'aventure vécue et pas complètement reflétée dans l'œuvre. Ce qui révèle que le percept a une dimension psychologique en même temps que sociale-idiomatique.

La valeur sémantique et poétique des langues se porte garante de la possibilité d'une certaine invariance du percept dans l'équivalence ou, pour être plus exact, dans la corrélation des messages redits en d'autres langues. Mais on ne peut pas oublier que la «perception» aboutit toujours à une expérience individuelle. Personne n'a le droit d'exiger de la traduction qu'elle recouvre la signification idiomatique en tant que figuration des choses ou manières de voir le monde, parce qu'elle est diverse, de même qu'on ne saurait lui demander de racheter des vécus et des expériences qui sont uniques parce qu'individuelles.

En fin de compte, c'est la culture, les connaissances partagées et la conviction intime d'une grande ressemblance psychologique entre les hommes qui font de la traduction un moyen privilégié de toute communication.

Ce percept total doit s'appréhender à travers le réseau signifiant qui se constitue comme ça en véhicule qui déclenche et conduit les compétences langagières et les intuitions poétiques par l'entremise de toute une série d'hypothèses portant sur le dire intenté et sur sa conformation ou mise en parole. L'espace formel extérieur n'est que l'idéogramme, le symbole très complexe des moments vécus de la conformation.

Tout porte à croire qu'il y a dans la textologie et dans l'histoire de l'écriture assez de confusions et de malentendus. Qu'est-ce que la forme du poème? Quel est son contenu? Questions générales qui méritent une réponse, mais que faisons-nous d'autres questions qui portent sur la forme du signifié ou du contenu conceptuel par opposition à la forme du signifiant et du contenu poétique ? C'est vouloir boucler la boucle systématique philosophiquement abstraite. On répondrait volontiers que tout est forme et que tout est contenu, car la forme a tout son contenu, de même que le contenu a toute sa forme, et pour sortir de ce cercle vicieux il faut ajouter que ce qui compte après tout est le message total et que le message présuppose intention et destinataire.

En effet, on a l'impression qu'on continue à confondre le texte comme un objet à tiroirs avec le texte-acte de parole, stratégie et enjeu de discours que l'écriture ne fait que figurer et symboliser. Il semble qu'on continue de se laisser leurrer par une matérialité du signe et par une systématicité «abstrayante». L'ennui est qu'on prétend combiner cette démarche philologique avec de nouveaux concepts tels que celui de contexte, de situations, de relation entre émetteur-destinataire, de référence, terminologie qui n'appartient pas réellement à une sémiotique, mais à une tout autre théorie de la parole vivante et communicante.

On a beau finalement vouloir s'entêter, et s'attarder, à ces approches positivistes du texte-objet, la vérité est que le texte n'est qu'un signal de signaux et que tout se passe dans et par le cerveau. Le texte n'est qu'un réseau symbolique dont on peut extraire des «représentations» de parole de l'imaginaire et de l'imaginé. L'écrit est le négatif d'une substance sonore qui ne fait qu'évoquer des expériences, ou des concepts, ou des images ou des sentiments.

La forme authentique est mentale et le contenu véritable est intentionnel, ce sont des objets de la conscience qui se sert de la connaissance, de la mémoire, du goût et de la jouissance esthétiques. Les acquis sont toujours le résultat de la compréhension globale d'une manière de dire qui dit «percepts». La parole est cette manière de dire : la forme, parce qu'elle est la seule à pouvoir se matérialiser dans une substance saisissable par les sens.

Mais cette forme renferme images et significations, réferences et intentions, concepts et sentiments, rythmes et émotivité, et tout ce qui constitue ce que nous avons 
défini comme le Sens. Tout texte (écrit) «figure» un discours (parlé) qui est une forme porteuse de sens, ou plutôt qui est un acte de conformation d'un sens. Voilà la révolution copernicienne à laquelle nous assistons et qu'il faut assumer sans crainte et sans regret.

Une fois adopté ce point de vue, notre attitude la plus intelligente consistera à chercher les paramètres ou registres de la forme poétique, de la conformation structurelle profonde à travers la double grille du réseau signifiant matériel et de sa figuration mentale, qui nous mèneront pas à pas à la quête du sens.

Parmi ces paramètres on pourrait discerner un stylistique-figuratif-imaginaire, un autre rhétorique-syntaxique-structural, un autre sémantique-lexical-symbolique, pas forcément dans cet ordre ni combinés de la sorte, et un autre rythmique-orchestral. Et c'est à ce dernier que je vais essayer de m'attaquer dans ce poème concret d'Yves Bonnefoy.

Pour commencer, je dirai que cette composition démontre que la Métrique ne pourra pas être tenue désormais pour le Patron rythmique. Cette manière de poétiser révèle par exemple ce qu'on n'a pas encore voulu comprendre, à savoir que le $e$ caduc ne pouvait plus, une fois disparu du parler, servir de mesure syllabique passe-partout pour le parfait décompte. De même pour l'anacrouse, la catalepse et ces périodes rythmiques mathématisantes et musicales.

Comment savoir maintenant s'il s'agit d'alexandrins ou d'hendécasyllabes ou d'ennéasyllabes ou d'octosyllabes? Les traducteurs de poèmes métriques se sont sans aucun doute aperçus bien avant les poètes iconoclastes du peu d'importance de ces exactitudes, même si les plus experts avaient certains préjugés de métrique comparée qui n'avaient jamais été soumis à l'épreuve.

Avec l'abandon, total ou partiel, des systèmes strophiques, on en vient à redécouvrir que le lieu de la poésie n'est autre que la prose, mais une prose fortement structurée et hautement rythmique où la rime est omniprésente et où le rythme orchestral est fait non seulement d'un composant syllabique mais d'un autre accentuel, syntaxique, tonal, intensif-symbolique et prosodique, et tout ça en même temps et unitairement.

Le traducteur qui connait bien la langue de départ n'a guère besoin que de deux ou trois lectures attentives pour appréhender le vouloir-dire conceptuel. Cependant l'attention se dirige dès les premières lectures sur certains mots et métaphores qui font problème. Qu'est-ce que veut dire «la pourvoyeuse du ciel noir»? Le traducteur prudent se dit que dans le cas des tropes il conviendra de ne pas se risquer et de recourir au calque.

D'autres fois il s'impose le choix quand il faut résoudre une ambiguité. Ainsi «Notre cœur» (ligne 8) serait en espagnol : ansia, afán, encono, même rabia, tandis que «ce cour» (1.12) signifierait inclinación, impulso, apetito, ou simplement corazón.

Sur le plan rythmique on constate, de prime abord, une unité séquentielle à dominante tétrasyllabique qu'il faudra traduire par une autre unité fonctionnellement corrélative. Ce biorythme propre à l'auteur justifie la lecture ou omission de certains $e$, mais il ne faut pas se méprendre sur les séquences à contraste qui sont ces quelques unités hexasyllabiques ou pentasyllabiques ou même trisyllabiques. Si l'on accorde au savoir-faire poétique de Bonnefoy ou à son instinct une certaine imprompte de la tradition métrique, on pourrait dire que le poème tend, au début, aux mesures décasyllabiques pour passer tôt et pleinement au dodécasyllabisme de l'alexandrin.

Cependant le «vers» 7 semble un hendécasyllabe et le 10 serait un alexandrin à la condition qu'il compte sur une coupe lyrique dans «la menteu/se», quoiqu'il serait plus exact de penser à une volontaire «cassure» du vers comme unité et noter un rejet de la syllabe RE du verbe «dire» qui rend possible une autre séquence tétrasyllabique: «De t'humilier/parmi tes lar/mes, de te di/re La menteu/se...»

Dans les vers centraux qui initient la seconde partie du poème, le rythme change visiblement: «Notre haut désespoir/sera que tu vi/ves, notre cœur que tu souf/fres». Là, 
l'intention d'un changement est évidente et il sera bon de se demander pourquoi, même si une simple traduction ajustée résout les problèmes.

La première unité séquentielle du poème, «Celle qui ruine 1'ê/tre», devrait constituer un hexasyllabe si on considère l'importance que l'idée métrique continue d'exercer, malgré les ruptures, mais il pourrait se lire comme un pentasyllabe. Ce n'est pas un cas isolé dans une ambiguité qui touche aussi aux hestiches: «sera que tu vi/ves» et à «sera suppliciée». Un critère personnel me pousse à compter cinq syllabes dans le premier cas et six dans le troisième, supposée une diérèse en «suppliciée».

Au fur et à mesure que le traducteur fait ses premières incursions conceptuelles et syntaxiques-rhétoriques-stylistiques, il s'arrête instinctivement aux difficultés que lui posera la structure rythmique, probablement parce qu'il se doute que ce niveau va être le plus difficile à imiter.

Néanmoins, la traduction n'étant finalement qu'un art de l'approximation et par là de la recréation, on établira que la forme du poème de Bonnefoy est pour l'essentiel cette régularité faite de martèlements et de brièvetés qui expriment la solennité, la rage et le mépris. Voilà le fondement premier, le plus profond substrat de cette structuration, que l'on se doit de transférer. La mise en œuvre, le fait que l'unité séquentielle se résume dans une mesure tétrasyllabique, que les voyelles accentuées aux ictus soient d'un côté le $i$ et le $e$, et de l'autre le $o$, le $u$ et le $a$, et que ce soient les consonnes liquides et dentales jointes aux labiales et aux sifflantes, celles qui réussissent à exprimer ce dédain tragique et la condamnation dramatique (le rythme de la syntaxe justifiant et confirmant ce rythme prosodique), révèle et rehausse la poétique de l'auteur, son style qui a su épouser si magistralement le contenu.

Cette trouvaille, cette accommodation du rythme au message, implique un deuxième degré d'«essentialité» que le traducteur aura à recréer, et je crois qu'entre langues sœurs cela est en grande partie possible. Il ne s'agirait pourtant pas de reproduire en détail chaque unité séquentielle ni chaque modulation pausale ou accentuelle - cela supposerait une copie impossible - mais d'en être conscient à tout moment et de savoir évaluer raisonnablement les gains et les pertes. En tout cas le degré d'approximation dépendra de la capacité expressive de la langue d'arrivée, à peine de sa sémantique, puisqu'on ne traduit pas uniquement les signifiés mais le sens et la coloration des messages.

Il n'est pas possible d'exposer dans les limites de cette étude les apports différents et parfois contraires que pourraient offrir à un modèle valable de structuration rythmique des sciences poétiques telles que la métrique du vers, la métrique du discours poétique ou la rythmique du sens. H. Meschonnic a essayé d'y apporter un peu de lumière ${ }^{3}$ et je me bornerai pour l'instant à inviter le lecteur intéressé à la lecture attentive de ses érudites, abondantes et judicieuses méditations.

Entre-temps je citerai volontiers un passage de son chapitre qui me semble justifier le bien-fondé initial du modèle que je proposerai pour la notation de la poésie française, modèle ouvert à d'autres poétiques et bien entendu, à d'autres critiques. «Le syllabisme seul - assure Meschonnic - ne peut être un modificateur structurel (c'est un démenti à l'idée d'un métricien du discours poétique aussi avisé que Bernard de Cornulier), pour la raison qu'il n'y a pas, et ne peut pas y avoir, en français au moins, de syllabisme seul. Le syllabisme n'apparaît qu'en rapport avec des groupes de sens, qui sont des groupes rythmiques, qui sont donc des groupes d'accent» ${ }^{4}$.

Cette argumentation suggère précisement l'importance fondamentale d'une première unité séquentielle syllabique et accentuée. Cette unité est cellule du rythme dans la mesure où elle réinstaure un retour de régularité qui a la «dominance». Mais il ne faut pas oublier les autres registres ni surtout le fait décisif: leur «complémentarité». Cet 
ensemble d'unités forme le leitmotiv essentiel d'une totalité orchestrale qui doit avoir une contrepartie ou contre-dominance dans chacun des niveaux et unités.

On pourrait sans aucun doute imaginer et ajouter de nouveaux registres, par exemple celui de l'«intensivité», qui serait caractérisé par une mise en relief de l'ictus lexical, du symbolisme sémantique de certains mots clés, mais ce qui compte c'est une coïncidence ou la rupture susceptibles d'être expliquées unitairement et avec la cohérence que peut fournir le sens. Il y aurait encore à ajouter que l'accent de groupe - non de mot qui caractérise le français dans le parler ordinaire et qui possède un sens mi-logique et mi-grammatical, est assumé par, et submergé dans, les unités syllabiques-tonales et dans les lexicales-intensives.

Il ne serait ni raisonnable ni pertinent d'établir une quelconque hiérarchie entre les registres cités ci-dessus, surtout parce que cela impliquerait une récidive dans l'apriorisme systématisant dont nous avons qualifié l'application d'erreur historique et d'imposture. D'ailleurs, quand nous tenons de préférence à l'étude de la structure purement rythmique nous ne voulons pas le moins du monde passer sous silence les autres composantes de la parole textuelle. Bien au contraire, nous souhaitons que les spécialistes réorientent leurs recherches sur une voie rentable et convaincante.

Quelques auteurs ne voulant plus de l'appareil symbolique de notation imposé par les métriciens traditionnels ont essayé d'esquisser des schémas nouveaux. C'est le cas de Meschonnic, qui est dans ce domaine un courageux et prestigieux pionnier. Après avoir signalé dans des études antérieures que sa manière de noter ne me convainquait pas tout à fait, je me devais, en toute justice, d'apporter des formulations alternatives.

Pour commencer on pourrait utiliser à la place des barres traditionnelles (simples ou doubles) symbolisant respectivement les coupes et les césures, une combinaison plus conforme à la véritable complexité rythmique. Voyons deux variantes du modèle proposé au critique :

$$
\begin{aligned}
& \overline{\text { Celle }} \overline{\text { qui }} \overline{\text { rui }} \overline{\text { ne }} \overline{\text { l'ề }}\left|\frac{1}{x}\right| \overline{\text { tre, }} \text { la } \overline{\text { beau té, }},\left|\frac{1}{x}\right| \\
& \overline{\text { Se }} \overline{\text { ra }} \overline{\text { sup }} \overline{\text { pli }} \overline{\text { ci }} \overline{\text { ée, }}\left|\frac{1}{\frac{1}{x}}\right|_{\text {mi }} \overline{\text { se }} \overline{\text { à }} \overline{\text { la }} \overline{\text { roue, }}\left|\frac{1}{x}\right|
\end{aligned}
$$

(Où la barre double signifierait la clôture syllabique, (') l'ictus accentuel et/ou lexicalintensif, (-), ( $($ ) les pauses — fortes et faibles — de la démarche mélodique et/ou syntaxique et $(\mathrm{x})$ les faîtes prosodiques).

$\mathrm{Au}$ lieu de la parenthèse il serait peut-être préférable de mettre en place d'autres symboles qui figurent la continuité:

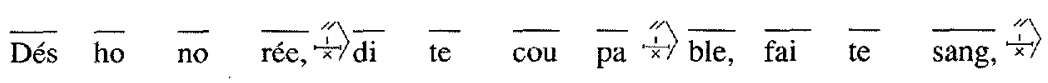

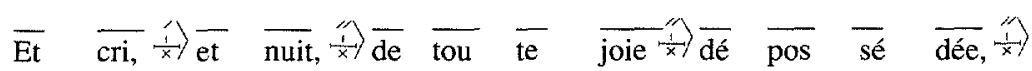

(Sur quoi on montrerait mieux l'impression d'une réelle continuité, même audétriment d'une économie scripturale. D'ailleurs, il y manque la signalisation des ictus de la «contre-dominance» qui me semble nécessaire à toute notation qui se respecte.) 
Mon intention n'est pas du tout d'imposer quoi que ce soit de définitif, mais bien au contraire d'offrir des voies, des esquisses possibles. S'il y avait à signaler tous les effets remarquables du rythme, il me semble qu'aucun système de notation qui dépasserait l'unité séquentielle et qui chercherait à rendre compte en plus du réseau prosodique ne serait ni économique, ni utile, ni même viable, car le rythme ne s'additionne pas seulement sur le cursus syntagmatique mais se multiplie plus ou moins «géométriquement» sur l'axe paradigmatique, la seule notation praticable en l'occurrence ne serait possible qu'au moyen d'un système numérique exponentiel qui ne saurait jamais, lui non plus, symboliser le véritable rythme de la croissance poétique.

Et puis, la première question qu'il faudrait se poser serait: Pourquoi la notation? Pour qui ? Si la réponse est: «pour le traducteur», on ne voit pas bien en quoi il serait indispensable de lui faire voir tous les effets acoustiques quand il est conscient qu'il ne sera pas capable de racheter une matière qui est essentiellement différente parce qu'idiomatique.

Uniquement entre langues søeurs, il est imaginable de mettre au point un «signestructe» corrélatif en langue d'arrivée qui soit élaboré très patiemment sous les présupposés d'une science telle que la phonostylistique qui a fait ses preuves depuis presque vingt ans. Ce travail est possible et je l'ai suggéré quand je parlais tout à l'heure de la combinaison vocalique et consonantique qui présidait à ce poème. Il n'y a pas lieu d'aller plus loin dans les limites de cette étude. Retraçons donc pour le moment la combinaison des ictus et des pauses, d'un côté, et de l'autre une amorce des variations qui constituent le nécessaire contrepoint rythmique et faisons-le au moyen d'un système traditionalisant qui tienne compte des barres et des contre-barres.

Je dois ajouter, avant de procéder à la notation, que je suis conscient de tout ce qu'elle a de discutable du fait même que je ne suis pas un expert en notations (ni en terminologie) musicales, ce qui assurerait par exemple un mieux-fondé des unités mélodiques et tonales.

Après tout, ce que le critique, et plus encore le traducteur qui est en fait le destinataire premier de cette tentative expérimentale, doit valoriser dans cet essai est la vraisemblance et la cohérence. Il y a là des signifiants symboliques moins démontrables — parce que plus subjectifs - que d'autres. Ils se réfèrent aux mouvements et aux items les moins objectivables.

Il n'y a là d'apprehendé que les grands traits, tour à tour expliqués et pressentis ou suggérés, un mouvement de baguette à quatre ou cinq compas qui ne doit pas faire oublier mais révéler bien des connaissances et des intentions de dire et des pesées de mots, et aussi bien des images et des pulsions et des reprises et des corrections et des accouplements qui vont tous, chez le poète, du vouloir-dire à l'expression et, chez le traducteur, de l'amas des hypothèses à partir de l'espace formel «mentalisé» à leur confirmation en sens compris et en réexpression collationnable.

Il y manque les autres composantes de la forme littéraire qui fondent aussi le message poétique. Bien des critiques et des poéticiens les ont traitées et une trêve s'impose peut-être pour leur reconduction sur des principes qui découlent d'une nouvelle théorie du texte. Le double apport - celui de la réflexion sur la théorie et celui d'une symbolisation des registres rythmiques - ne vise qu'à une continuation nécessaire. Mais que personne n'oublie que toute traduction requiert une seconde phase de reformulation, de récriture - tâche immense et délicate s'il en est — un projet de ré-invention et de réincarnation dans une autre langue de ce total percept, subjectif parce qu'interprété, provisoire parce qu'ouvert à bien des lectures, dont la réussite, toujours partielle, n'a d'autre garantie que cette démarche intelligente et sentie du processus interprétatif. Voilà, pour finir, le schéma de notations que je propose: 


\section{Tableau de notations :}

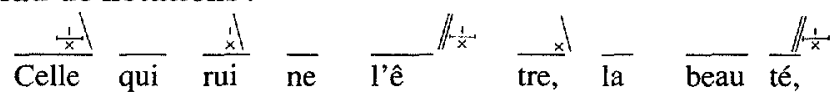

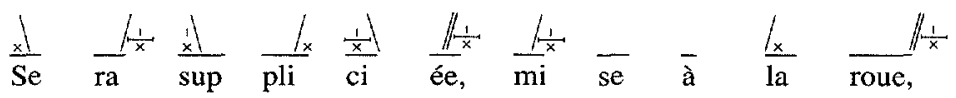

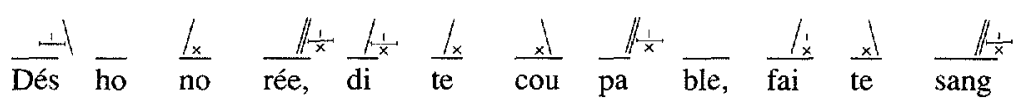

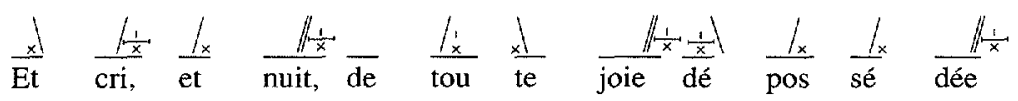

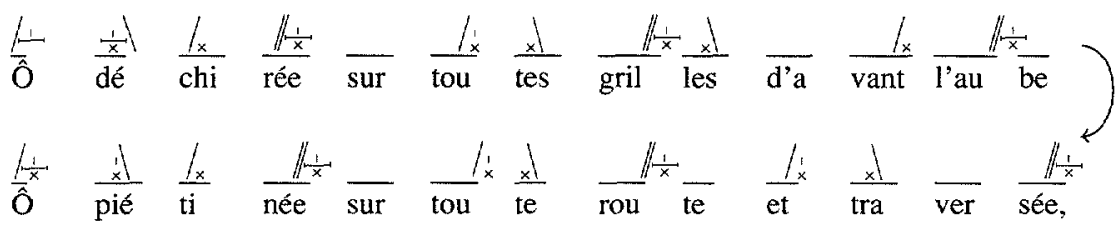

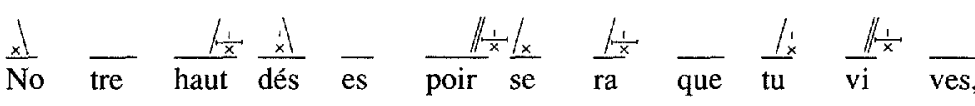

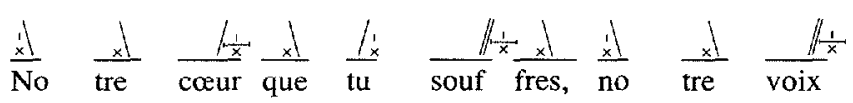

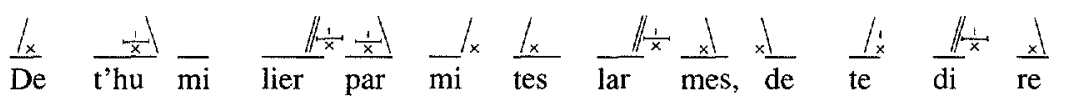

$\frac{l x}{\text { La }} \frac{x \mid}{\operatorname{men}} \frac{\| \frac{1}{x}}{\text { teu }} \frac{x \mid}{\text { se, }} \quad \frac{\mid x}{\text { la }} \frac{x \mid}{\text { pour }} \frac{1}{\text { vo }} \frac{\mid \frac{1}{x}}{\text { yeu }} \frac{x \mid}{\text { se }} \frac{x \mid}{\text { du }} \frac{l_{x}}{\text { ciel }} \frac{\| \frac{1}{n}}{\text { noir, }}$

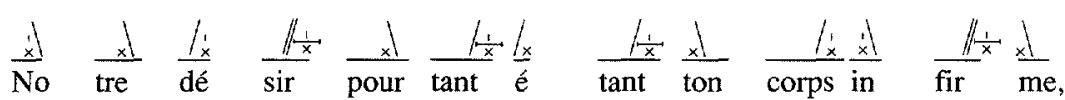

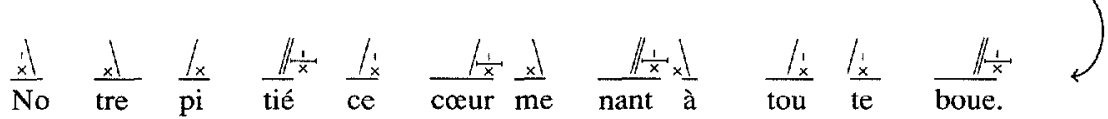




\section{NOTES :}

1. Cette composition appartient au recueil Hier régnant désert, publié en 1958. Cette même année Yves Bonnefoy a expliqué - dans «L'acte et le lieu de la poésie» - sa vision de l'histoire de cet art en France surtout à partir de Baudelaire et de sa poétique moderne. Pour les détails, voir l'ouvrage d'Yves Bonnefoy: Du mouvement et de l'immobilité du Douve, suivi de Hier régnant désert, et accompagné d'Anti-Platon et deux autres essais, NRF, «Poésie / Gallimard», Mercure de France, 1970, p. 153.

2. En ce sens, et pour une information plus détaillée je suggère la lecture de mon recueil d'articles récemment paru sous le titre Percepto mental y estructura rítmica, publié sous le patronage de l'Université d’Extremadure et imprimé à Salamanque (Europa Artes Gráficas, novembre 1987).

3. Voir son livre Critique du rythme: Anthropologie historique du langage (chap. $\mathrm{X}$ : «Métrique pure ou métrique du discours», p. 519-562), Paris, Verdier, 1982.

4. Voir p. 561-562. La réflexion mise entre parenthèses est de moi. L'ouvrage de Bernard de Cornulier, que Meschonnic connaît et cite parmi des centaines d'autres des langues poétiques les plus diverses, est sa thèse Problèmes de métrique française, soutenue à l'Université de Provence le 29 juin 1979, 2 tomes, 13009 Marseille, éditions Janine Tesseire, Faculté des Sciences de Luminy. Elle devait parâtre aux éditions du Seuil. 